\title{
Evaluation of a new artificial tear formulation for the management of tear film stability and visual function in patients with dry eye
}

This article was published in the following Dove Press journal:

Clinical Ophthalmology

19 October 2017

Number of times this article has been viewed

\author{
Gail Torkildsen' \\ Mile Brujic ${ }^{2}$ \\ Michael S Cooper ${ }^{3}$ \\ Paul Karpecki ${ }^{4}$ \\ Parag Majmudar ${ }^{5}$ \\ William Trattler 6 \\ Meryl Reis ${ }^{7}$ \\ Joseph B Ciolinol,8
}

'Andover Eye Clinic, Andover MA, ${ }^{2}$ Premier Vision Group, Bowling Green, $\mathrm{OH},{ }^{3}$ Windam Eye Group, Willimantic, CT, ${ }^{4}$ Gaddie Eye Center, Louisville KY, ${ }^{5}$ Chicago Cornea Consultants, Chicago IL, ${ }^{6}$ Center For Excellence in Eye Care, Miami, FL, ${ }^{7}$ Rohto Mentholatum Research Laboratories, Horsham, PA, ${ }^{8}$ Massachusetts Eye and Ear Infirmary, Boston MA, USA
Correspondence: Gail Torkildsen 300 Brickstone Square, Andover, MA 01810 , USA

Tel +l 9786858900

Email mdlasik@gmail.com
Purpose: Artificial tears are the first line of therapy for dry eye disease (DED) and are also the most frequently used treatment approach for this common condition. Despite this, there are few published studies that directly compare the effectiveness of different drop preparations, especially those formulated specifically for dry eye. In this study, we tested a new artificial tear product, Rohto ${ }^{\circledR}$ Dry-Aid ${ }^{\mathrm{TM}}$, for its ability to relieve the signs and symptoms of DED. The study used a second drop, Systane ${ }^{\circledR}$ Ultra, as a positive comparator.

Materials and methods: This was a prospective, single-center, open-label, parallel-group study comparing the effects of the two products when used continuously over $\sim 30$ days (Clinical Trials registration number NCT03183089). Subjects were randomly assigned to one of the two test groups and were monitored 2 and 4 weeks after enrollment. Efficacy endpoints included ocular staining, visual function, and ocular discomfort.

Results: Treatment groups had similar ocular staining and ocular comfort scores, and both showed statistically significant ocular discomfort score improvement. Subjects in the Rohto group reported significant improvements in visual tasking activities such as watching television and driving at night. There was also a tendency for diary symptom scores to worsen from morning to evening in the Systane group, but not in the Rohto group; this trend was not significant, but warrants further study.

Conclusion: The two products, Rohto Dry-Aid and Systane Ultra, elicited comparable effects on the signs and symptoms of DED. While both products are designed to provide long-lasting relief, subjects in the Rohto group experienced a superior relief from discomfort associated with visual tasking activities and daily diaries, indicating that the Rohto drops may provide a longer duration of symptomatic relief over the course of the day.

Keywords: artificial tears, dry eye, ocular surface, visual tasking, blurred vision

\section{Introduction}

Dry eye disease (DED) is the most prevalent form of ocular discomfort and irritation, with estimates of affected individuals ranging from 1 in 20 to as high as 1 in every 5 adults in the USA who experience some degree of mild to moderate dry eye. ${ }^{1,2}$ Individuals with DED represent a broad spectrum of the population: it is common among postmenopausal women and in those who have undergone ocular procedures such as LASIK or cataract removal; it can be due to autoimmune conditions (such as Sjögren's syndrome, systemic lupus erythematosus, diabetes, thyroid disease, or rheumatoid arthritis) or can be brought on by long-term contact lens use. ${ }^{3,4}$ The symptoms of DED including ocular burning, stinging, grittiness, and blurred vision can be exacerbated by environmental conditions such as low humidity, air-conditioned 
workplaces, winter heating, a dusty or windy outdoor environment, or prolonged computer use. ${ }^{5}$ Other factors, such as hormonal imbalance, systemic or topical medications, anatomic changes, ocular trauma, radiation therapy, and aging, can also contribute to DED. With the increased use of computers, cell phones, and other visual technologies, along with an aging population in the USA and in other countries, it is expected that DED prevalence will continue to increase in the years to come. ${ }^{1,6}$

In addition to its symptomology, DED is also associated with reduced tear production and an increase in tear film instability, both of which contribute to exposure of, and damage to, the ocular surface. ${ }^{7}$ The condition is frequently accompanied by inflammation of the ocular surface, ${ }^{8}$ although this is often poorly correlated with symptomatic DED. ${ }^{3,8}$ Currently, the most commonly used treatment for mild to moderate DED is tear substitutes or artificial tears. ${ }^{9}$ These formulations are most often comprised of a physiological saline with some mixture of surface lubricant formulated to supplement the natural constituents of the tear film.

The natural tear film is an extracellular fluid covering the epithelial cells of the corneal and conjunctival surfaces. This liquid coating is a critical medium for function, protection, and maintenance of the ocular surface. ${ }^{10,11}$ The tear film functions to lubricate the surface and the lids, to optimize the refractive function of the anterior segment, and to provide a means for removal of environmental contaminants from the ocular surface. The tear film is composed of three layers: an outer lipid layer $(\sim 0.1 \mu \mathrm{m}$ thick) produced by the meibomian glands in the tarsal plate, a central aqueous layer $(\sim 7-10 \mu \mathrm{m}$ thick) produced by both the main and accessory lacrimal glands, and an inner mucin layer $(\sim 0.2-1.0 \mu \mathrm{m}$ thick) produced by goblet cells in the conjunctiva. ${ }^{10-13}$ Tear substitutes are generally designed to augment this tear composition and to stabilize the compromised tear film of the DED patient.

Rohto Dry-Aid (Rohto; The Mentholatum Company, Orchard Park, NY, USA) is a lubricant eye drop compliant with the title 21 Code of Federal Regulations (CFR) monograph for over-the-counter ophthalmic demulcent eye drops. It is a sterile, buffered product packaged in a multidose container. The drops contain the active lubricants propylene glycol and povidone in a clear microemulsion formulation. According to the manufacturer, Rohto is designed to replicate the three layers of the tear film to provide both immediate and long-lasting relief of DED by creating a uniform lipid layer in a transparent drop. Systane Ultra (Systane; Alcon, Fort Worth, TX, USA) is one of the most widely used ocular lubricants in the USA. It combines polyethylene glycol with propylene glycol lubricants in an aqueous formulation with an HP-Guar demulcent designed to provide a prolonged lubrication to the ocular surface.

In this single-center, parallel-group, open-label study, the ability of Rohto and Systane to relieve the signs and symptoms of DED was compared over $\sim 30$ days in subjects diagnosed with mild to moderate DED. Subjects were assigned to one of the two products and were monitored at 2 and 4 weeks after enrollment. Efficacy endpoints included corneal fluorescein staining, lissamine staining, visual function tests, ocular discomfort (measured with the Ora Calibra ${ }^{\mathrm{TM}}$ Ocular Discomfort Scale and the Ora Calibra Ocular Discomfort \& 4-Symptom Questionnaire), diary discomfort scales, and the Ocular Surface Disease Index $\left(\mathrm{OSDI}^{\odot}\right)$ measurements.

Results were assessed based on comparisons to baseline measures and on direct comparisons between the two products at specified time points. A key to the study design was that it represented an assessment conducted in a reallife, real-time setting rather than a strictly clinic-based trial. Our goal was an objective comparison of the overall efficacy of a new over-the-counter drop to another product currently in common use, rather than to a placebo drop, where any comparative conclusions would be indirect. This approach is likely to provide clinicians a more useful measure of the relative effectiveness of these two dry eye therapies.

\section{Materials and methods}

This was a prospective, single-center, multivisit, parallelgroup study with a targeted enrollment of 80 subjects. Subjects were screened and those who met the eligibility criteria were randomized to one of two treatment groups, Rohto or Systane. All subjects received the test agent for a total of 30 days of twice a day (BID) dosing, and all were examined at two visits during the course of the study, at 15 and 30 days. Written informed consent was obtained from the subjects before any procedure specified by this protocol was initiated, including the screening procedures. The study protocol and informed consent forms were approved by the Alpha Institutional Review Board, and all study procedures and documents were performed and collected in accordance with International Committee on Harmonization guidelines.

\section{Inclusion/exclusion criteria}

Subjects (18 years of age or older) screened for the study were required to have a subject-reported history of dry eye for at least 6 months, including a history of use or desire to use eye drops for dry eye relief. They were required to meet minimum scores $(\geq 2)$ in at least one of the dry eye symptom 
assessments and one of the corneal staining measures ( $\geq 2$ in at least one region), and report an OSDI score of at least 13. They were also required to have an average tear film break-up time $($ TFBUT) $\leq 5$ seconds. All of these criteria needed to apply to at least one eye at the screening visit (Visit 1). In addition, subjects with significant slit-lamp findings at screening, or those who had any ongoing ocular infection (bacterial, viral, or fungal) or active ocular inflammation (eg, follicular conjunctivitis) were excluded. Similarly, those subjects who had worn contact lenses within 7 days of screening or anticipated using contact lenses during the study and those who had used any eye drops within 2 hours of screening were also excluded. Additional exclusion criteria included LASIK surgery within the last 12 months; use of Restasis ${ }^{\circledR}$ within 30 days of screening; current or planned use of any topical ophthalmic medications, gels, or scrubs during the study period; and unwillingness to discontinue these medications for the duration of the trial. Subjects with corrected visual acuity greater than or equal to $\log \mathrm{MAR}+0.7$ as assessed by the Early Treatment Diabetic Retinopathy Study scale in both eyes or those with an uncontrolled systemic disease were also excluded. In addition, patients with any condition that in the opinion of the investigator may have put the subject at significant risk, confounded the study results, or may have interfered significantly with the subject's participation in the study were also excluded. Women of childbearing age were screened for pregnancy and asked to use an appropriate form of birth control for the duration of the study. All subjects were required to avoid use of any investigational drug or device, and all were required to avoid any drugs known to cause ocular drying, unless these medications were used on a stable dosing regimen for at least 30 days prior to screening.

\section{Schedule of visits}

Subjects underwent a series of screening tests on the first day of the study (Visit 1, Day 1) to confirm that they met the inclusion criteria; these are summarized in Table 1. Screening included a medical and medication history, ocular discomfort assessments (including OSDI), visual acuity and visual function tests, TFBUT measurement, fluorescein staining, lissamine staining, and slit-lamp biomicroscopy. Screening results were used to identify subjects for randomization, who then completed a second series of assessments after instillation of the first dose of test compound. At the end of Visit 1,

Table I Schedule of visits and procedures ${ }^{\mathrm{a}}$

\begin{tabular}{|c|c|c|c|c|}
\hline \multirow[t]{2}{*}{ Procedures } & \multicolumn{2}{|c|}{ Visit I Day I } & \multirow[t]{2}{*}{ Visit 2 Day $15 \pm 2$} & \multirow[t]{2}{*}{ Visit 3 Day $29 \pm 2$} \\
\hline & Predosing & Postdosing & & \\
\hline Informed consent/HIPAA & $\mathrm{x}$ & & & \\
\hline Medical/medication history and demographic & $x$ & & & \\
\hline Study drug collection & & & $x$ & $x$ \\
\hline Diary collection & & & $x$ & $x$ \\
\hline Medical/medication history update & & & $x$ & $x$ \\
\hline Adverse event query & & & $x$ & $x$ \\
\hline Pregnancy test & $x^{b}$ & & & $x$ \\
\hline \multicolumn{5}{|l|}{ Ora Calibra ${ }^{\mathrm{TM}}$ symptom assessments } \\
\hline Ocular Discomfort Scale & $x$ & $X^{c, d}$ & $x$ & $x$ \\
\hline Ocular Discomfort and 4-Symptom Questionnaire & $x$ & $X^{c, d}$ & $x$ & $x$ \\
\hline Quality of Life Questionnaire & $x$ & & $x$ & $x$ \\
\hline $\mathrm{OSDI}^{\odot}$ questionnaire & $x$ & & $x$ & $x$ \\
\hline Visual acuity & $x$ & & $x$ & $x$ \\
\hline Visual function assessments & $x$ & $X^{d}$ & & $x$ \\
\hline Slit-lamp biomicroscopy & $x$ & & $x$ & $x$ \\
\hline TFBUT & $x$ & $X^{c, d}$ & $x$ & $x$ \\
\hline Fluorescein staining & $x$ & & $x$ & $x$ \\
\hline Lissamine green staining & $x$ & & $x$ & $x$ \\
\hline Review of qualification criteria & $x$ & & & \\
\hline Ora Calibra drop comfort scale and questionnaire & & $\mathrm{x}$ & & \\
\hline All day relief questionnaire & & & & $x$ \\
\hline Study drug dispensation & $x$ & & $x$ & \\
\hline Diary dispensation & & $x$ & $x$ & \\
\hline Exit subject from the study & & & & $x$ \\
\hline
\end{tabular}

Notes: a Some clinical measures that showed no significant differences between groups have been omitted for clarity. ${ }^{\mathrm{b}}$ For females of childbearing potential. ${ }^{\mathrm{c}}$ Measurements performed at 5 and 20 minutes post-instillation at Visit I. Measurements performed at 60 minutes post-instillation at Visit I.

Abbreviations: HIPAA, Health Insurance Portability and Accountability Act; OSDI, Ocular Surface Disease Index; TFBUT, tear film break-up time. 
subjects were provided sufficient test compound for 2 weeks of BID dosing and were also provided a diary along with instructions to record symptoms twice daily, morning and evening, prior to drop instillation. At the 2-week follow-up (Visit 2, Day 15 \pm 2 ), diaries and remaining test agents were collected, and subjects were provided replacements for both. All measures conducted at Visit 1 (except visual function tests) were repeated, and subjects were instructed to continue BID dosing and diary entries for an additional 2 weeks.

At the 4-week follow-up (Visit 3, Day 29 \pm 2 ), diaries and remaining test agents were collected, and a subject medical history update was acquired. Pregnancy tests were repeated, and any adverse events were noted. All measures conducted at Visit 1 were repeated, and subjects completed diary entries and then exited from the study.

\section{Statistical methods}

The continuous and ordinal efficacy variables collected at each visit were summarized statistically and analyzed using two-sample $t$-tests comparing Rohto to Systane. Comparisons of the continuous and ordinal efficacy variables with baseline within the Rohto group were analyzed using a paired $t$-test.

\section{Results}

A total of 80 subjects were enrolled in the study, and all these subjects completed the entire 30-day duration. Demographics of the two study groups were statistically comparable (Table 2). As part of the initial assessments, subjects were asked to rate immediate post-instillation drop comfort using a $0-10$ point scale, where 0 is the most comfortable, and both products scored in the very comfortable range (2.05 vs 1.96 , $p=0.766$ ). The ocular comfort scores also confirmed that both products provided an immediate, significant improvement in ocular comfort that was sustained for at least 1 hour after instillation (Table 3).

Diminished tear film and associated inflammation can lead to a compromised ocular surface; signs of this aspect of DED include increases in corneal fluorescein staining and conjunctival hyperemia. Mean values for fluorescein staining are shown in Table 4. Both agents appeared to yield modest improvement in staining for most regions, particularly at Visit 2. Scores for

Table 2 Enrolled subject demographics

\begin{tabular}{lllll}
\hline & Total & Male & Female & Mean age \\
\hline Rohto Dry-Aid & 39 & 13 & 26 & 61 \\
Systane Ultra & 41 & 16 & 25 & 62 \\
Total & 80 & $29(36 \%)$ & $51(64 \%)$ & 62 \\
\hline
\end{tabular}

conjunctival hyperemia (not shown) also exhibited similar modest improvement in the measures at Visits 1 and 2.

Another well-characterized sign of DED is reduced tear film stability, as measured by the TFBUT. When stained with a fluorescein dye, the integrity of the tear film can be observed as the time (in seconds) from a blink to the disruption of the tear film layer. Dry eye is associated with decreased TFBUT. Both test agents elicited increases in TFBUT that were sustained for the 60-minute assessment window at Visit 1 (Table 5). These increases were statistically significant for both test agents at 5 minutes post-instillation; the significance of the increase was preserved for the Rohto group at 20 and 60 minutes post-instillation.

Ocular discomfort was measured in several ways over the course of the study. At the study visits, subject discomfort was measured using the Ora Calibra Ocular Discomfort Scale and the Ora Calibra Ocular Discomfort \& 4-Symptom Questionnaire. This scale asks patients to grade discomfort along with burning, drying, grittiness, and stinging on a 0-4 scale. Subjects in both test groups reported reduced discomfort scores for all symptoms that were sustained over subsequent study visits. Mean values for these measures are presented in Table 6. Both groups displayed similar mean values at Visit 1 , and measures for all symptoms improved after instillation of test agents and remained below the initial values over the course of the study. One symptom score, grittiness, displayed a significant improvement in the Rohto group when compared to subjects in the Systane group at Visit 1.

Subjects were also queried using the Ora Calibra Quality of Life Questionnaire at Visit 1 (predosing) and again at Visits 2 and 3. The Quality of Life Questionnaire asks subjects to respond to four questions (using a $0-4$ scale) focusing on the impact of their DED on everyday visual tasking; questions such as "how much has your eyesight interfered with your daily activities this past week?" and "have you been troubled by eye problems while reading, watching television, or driving at night?" were asked. These questions focused on the issues most DED subjects raise when discussing the impact of their dry eye on daily living. Mean values for responses at Visits 2 and 3 were compared with those at Visit 1 to assess the impact of test agents on the quality of life (Table 7). Subjects in both test groups reported significant improvement (ie, decreased mean scores) in how much their eyesight "interfered with daily activities" at Visit 3; subjects in the Rohto group showed significant improvement at Visit 2 as well. Subjects in the Rohto group also reported significant improvement in the degree to which their DED impacted television or movie viewing and how 
Table 3 Ocular comfort scores

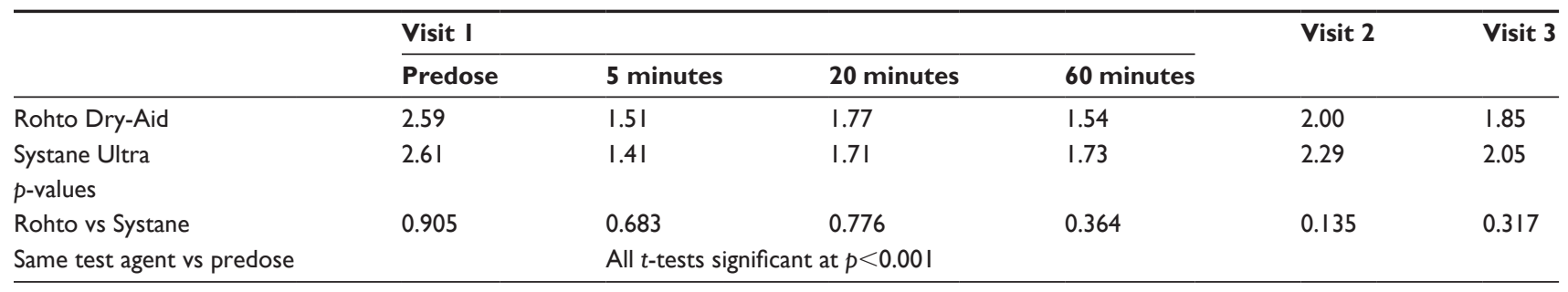

Notes: Subjects were asked to score their level of discomfort using the Ora Calibra Ocular Discomfort Scale ( $0-4$, where 0 is no discomfort and 4 is constant discomfort) prior to drop instillation and at 5, 20, and 60 minutes after instillation at Visit I; single measures were collected at Visits 2 and 3.

much it impacted driving at night. Improvements in these quality-of-life measures for the Systane group were not significant (Table 7).

Data using the Ora Calibra Ocular Discomfort \& 4-Symptom Questionnaire were also collected with the daily diary scores, where subjects provided a numerical value for discomfort and for four symptoms as part of their BID diary of dry eye symptomology. There were no statistically significant differences between treatment groups in the mean values of diary scores. Despite this, it is interesting to note that, especially for ocular discomfort and dryness, Rohto mean scores were approximately the same from morning to evening, while Systane mean scores were trending upward from morning to evening. Differences in all of the mean scores for discomfort and for the four symptoms between morning and evening are shown graphically in Figure 1. In four of the five measures, scores for Rohto were flat, with no more than $\sim 2 \%$ change from morning to evening. The exception is grittiness, where the mean scores declined by $>10 \%$. In contrast, four of five mean scores for Systane increased from $5 \%$ to $18 \%$.

\section{Discussion}

The objective of this study was to evaluate the efficacy of two artificial tear products, Rohto Dry-Aid and Systane Ultra, for treatment of ocular signs, symptoms, and visual

Table 4 Mean corneal fluorescein staining by visit

\begin{tabular}{llllll}
\hline & Inferior & Superior & Central & Temporal & Nasal \\
\hline Rohto Dry-Aid & & & & & \\
$\quad$ Visit I & 2.04 & 2.27 & 0.97 & 1.92 & 1.96 \\
Visit 2 & 2.09 & 1.86 & 0.88 & 1.82 & 1.86 \\
$\quad$ Visit 3 & 2.23 & 2.15 & 1.14 & 2.15 & 2.21 \\
Systane Ultra & & & & & \\
Visit I & 1.93 & 2.01 & 0.79 & 1.63 & 1.80 \\
Visit 2 & 1.66 & 1.57 & 0.59 & 1.43 & 1.46 \\
Visit 3 & 2.07 & 1.96 & 0.91 & 1.88 & 1.89 \\
\hline
\end{tabular}

Notes: Scores listed for the worst eye, which was defined as the eye with the highest staining score in inferior, superior, or central regions at Visit I. Both Rohto and Systane groups exhibited modest improvement in staining scores. function issues in subjects diagnosed with DED. Subjects were assessed for measures of DED over a 4-week period, during which they self-administered BID treatment with one of the two test agents. In all of these assessments, the efficacy of the two agents was comparable. Ocular staining, tear film metrics, and visual function tests all exhibited modest improvement in subjects from both test groups. In the one measure where it was possible to draw a clear distinction, improvements in three of four quality-of-life metrics were significantly greater for the Rohto users after 4 weeks of BID dosing.

The hypotheses tested in this study were twofold. The first hypothesis was that the Rohto improved visual function and ocular surface staining in DED patients. While not all metrics reached the level of statistical significance, Rohto showed a consistent ability to improve the subject signs and symptoms. The second hypothesis was that the effect of Rohto on the signs and symptoms of DED was equal to or greater than the effect seen with Systane. In no case was there a significant difference between the two test treatments that favored Systane; Rohto demonstrated an ability to alleviate the signs and symptoms of DED that was, by all measures, comparable to that of Systane, with a positive overall use experience.

One of the most intriguing distinctions observed between the two test agents was in diary data (Figure 1) that showed an increase in symptomology over the course of the day (AM

Table 5 Tear film break-up time

\begin{tabular}{|c|c|c|c|c|}
\hline & \multicolumn{4}{|l|}{ Visit I } \\
\hline & Predose & 5 minutes & 20 minutes & 60 minutes \\
\hline Rohto Dry-Aid & 2.44 & 3.32 & 3.84 & 3.27 \\
\hline$p$-value vs baseline & & 0.011 & 0.006 & 0.012 \\
\hline Systane Ultra & 2.27 & 3.09 & 2.87 & 2.75 \\
\hline$p$-value vs baseline & & 0.018 & 0.206 & 0.442 \\
\hline
\end{tabular}

Notes: Mean break-up time, in seconds, scored for the worst eye only; $p$-values vs baseline. Mean values were significantly increased for both groups at 5 minutes, but the mean scores of the Rohto group only maintained the increased break-up time at 20 and 60 minutes. $p$-values in bold indicate significance $(p<0.05)$. 
Table 6 Ora Calibra Ocular Discomfort and 4-Symptom Questionnaire

\begin{tabular}{|c|c|c|c|c|c|c|c|}
\hline & \begin{tabular}{|l} 
Rohto \\
Dry-Aid
\end{tabular} & $\begin{array}{l}\text { Systane } \\
\text { Ultra }\end{array}$ & $\begin{array}{l}\text { Ocular } \\
\text { Discomfort }\end{array}$ & Burning & Dryness & Grittiness & Stinging \\
\hline \multirow{3}{*}{$\begin{array}{l}\text { Visit I } \\
\text { pre-dosing }\end{array}$} & Rohto & & 2.95 & 1.64 & 3.21 & 1.67 & 1.18 \\
\hline & & Systane & 2.83 & 1.49 & 2.90 & 2.05 & 1.24 \\
\hline & \multicolumn{2}{|c|}{$p$-value } & 0.478 & 0.593 & 0.114 & 0.197 & 0.815 \\
\hline \multirow{3}{*}{$\begin{array}{l}\text { Visit I } 5 \text { Min } \\
\text { Post-Dose }\end{array}$} & Rohto & & 1.74 & 0.90 & 1.54 & 0.59 & 0.82 \\
\hline & & Systane & 1.68 & 0.80 & 1.68 & 1.27 & 1.05 \\
\hline & \multicolumn{2}{|c|}{$p$-value } & 0.815 & 0.698 & 0.599 & 0.012 & 0.374 \\
\hline \multirow[t]{3}{*}{ Visit 2} & Rohto & & 2.23 & 1.31 & 2.26 & 1.15 & 0.85 \\
\hline & & Systane & 2.17 & 1.17 & 2.20 & 1.63 & 0.85 \\
\hline & \multicolumn{2}{|c|}{$p$-value } & 0.772 & 0.594 & 0.790 & 0.062 & 0.972 \\
\hline \multirow[t]{3}{*}{ Visit 3} & Rohto & & 1.97 & 0.97 & 2.15 & 1.36 & 0.79 \\
\hline & & Systane & 2.15 & 1.22 & 2.29 & 1.59 & 0.85 \\
\hline & \multicolumn{2}{|c|}{$p$-value } & 0.420 & $0.34 I$ & 0.519 & 0.386 & 0.802 \\
\hline
\end{tabular}

Notes: Subjects rated the severity of each of the following symptoms with regards to how both their eyes felt, in general: overall ocular discomfort, burning, dryness, grittiness, and stinging, according to a 6 -point $(0-5)$ scale, where $0=$ none and $5=$ worst. Scores were collected before and after dosing at Visit $I$ and at Visits 2 and 3 . Both agents reduced mean discomfort scores postdosing at Visit I; the decrease in mean grittiness score in the Rohto group was significantly greater than that seen in the Systane group. $p$-values in bold indicate significance $(p<0.05)$.

Table 7 Ora Calibra ${ }^{\mathrm{TM}}$ Quality of Life Questionnaire

\begin{tabular}{|c|c|c|c|}
\hline \multirow[t]{2}{*}{ In the past week } & Visit I & Visit 2 & Visit 3 \\
\hline & \multicolumn{3}{|c|}{ Mean score ( $p$-value vs Visit I) } \\
\hline \multicolumn{4}{|c|}{ How much has your eyesight impacted your daily activity? } \\
\hline Rohto & 1.08 & $0.77(0.012)$ & $0.74(\mathbf{0 . 0 1 4})$ \\
\hline Systane & 1.05 & $0.93(0.281)$ & $0.73(0.036)$ \\
\hline \multicolumn{4}{|c|}{ How troubled have you been reading at night? } \\
\hline Rohto & 1.54 & $1.56(0.893)$ & $1.77(0.270)$ \\
\hline Systane & 1.78 & $1.88(0.660)$ & I.7I (0.734) \\
\hline \multicolumn{4}{|c|}{ How troubled have you been watching television or movies at night? } \\
\hline Rohto & 1.31 & $1.0(0.123)$ & $0.85(\mathbf{0 . 0 1 6})$ \\
\hline Systane & 1.27 & $0.98(0.096)$ & $1.02(0.168)$ \\
\hline \multicolumn{4}{|c|}{ How troubled have you been driving at night? } \\
\hline Rohto & 1.85 & $1.62(0.284)$ & $1.44(0.034)$ \\
\hline Systane & 1.78 & I.7I (0.660) & $1.61(0.342)$ \\
\hline
\end{tabular}

Notes: Subjects were asked to indicate how troubled they have been during the prior week while performing specific visual tasks described in the questions, using a $0-4$ scale, where $0=$ minimal and $3=$ severe; a score of 4 indicated the question was not applicable. Scores were collected at Visits I, 2, and 3. Mean scores for three of the four questions were significantly reduced at Visit 2 in the Rohto group and in one of the four questions in the Systane group. $p$-values in bold indicate significance $(p<0.05)$.

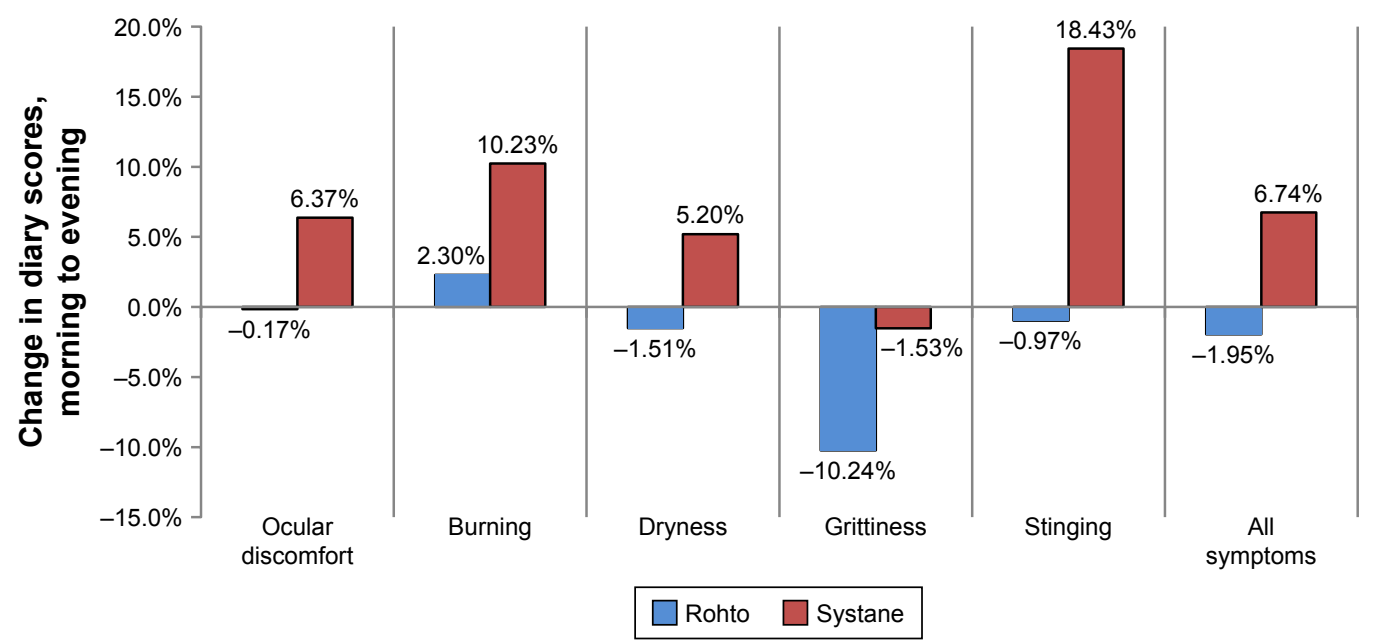

Figure I Self-reported symptom scores: comparison of mean AM vs PM scores.

Notes: Subjects used the Ora Calibra Ocular Discomfort \& 4-Symptom Questionnaire to rate their symptoms in a daily diary; ratings were collected in the morning and evening over the 4-week course of the study. Graph shows the percent change in mean scores between AM and the PM for each of the five symptoms. Positive values indicate a worsening of symptoms in the evening, which was observed for 4 of the 5 symptom scores for Systane. 
vs PM) for the Systane users, while the Rohto users' scores remained stable throughout the day. It is known that there are diurnal variations in the tear film, and it may be these variations that are responsible for the fluctuations in symptom scores for the two agents. ${ }^{7,13}$ Alternatively, the difference may be a reflection of differences in the composition of the two drops (a microemulsion with uniform lipid layer compared to an aqueous formulation with guar demulcent) that may result in performance differences. Such a difference is seen in the 20- and 60-minute post-instillation TFBUT, where the Rohto drop provided a prolonged stabilization of the tear film. This may also indicate a mechanism for the observed performance differences. It is likely that the distinctions between the two products reflect both differences in component actives as well as formulation excipients, as seen in comparisons of other artificial tear formulations. ${ }^{14}$ Further studies would be needed to confirm the observed distinctions between the two formulations.

\section{Acknowledgments}

Support of this study was provided by the Mentholatum Company, Orchard Park, NY, USA. Writing assistance provided by $\mathrm{J}$ McLaughlin of Ora, Inc.

\section{Disclosure}

Dr Torkildsen was clinical investigator for this study, and serves as clinical consultant at Ora, Inc. where the study was conducted. She also has received travel support from Alcon. Dr Majmudar has served as a consultant to the Mentholatum Company. Dr Brujic, Dr Cooper, Dr Karpecki, and Dr Trattler have served as consultants to Alcon and to the Mentholatum
Company. Ms Reis is an employee of Rohto Mentholatum Research Laboratories, a division of The Mentholatum Company. Dr Ciolino serves as a clinical consultant at Ora, Inc. and also has served as consultant to Alcon. The authors report no other conflicts of interest in this work.

\section{References}

1. Gayton JL. Etiology, prevalence, and treatment of dry eye disease. Clin Ophthal. 2009;3:405-412.

2. The epidemiology of dry eye disease: report of the Epidemiology Subcommittee of the International Dry Eye Work Shop. Ocul Surf. 2007;5(2):93-107.

3. Chong E, Harissi-Dagher M, Dana R. Wetting of the Ocular Surface and Dry-Eye Disorders. In: Albert \& Jakobiec's Principles and Practices of Ophthalmology 3rd ed. Canada: Elsevier; 2008:773-788.

4. Yu T, Shi WY, Song AP, Gao Y, Dang GF, Ding G. Changes of meibomian glands in patients with type 2 diabetes-mellitus. Int J Ophthalmol. 2016;9(12):1740-1744.

5. The International Dry-Eye Workshop (DEWS Report). The definition and classification of dry eye disease: report of the definition and classification subcommittee of the international dry eye workshop. Ocul Surf. 2007;5(2):75-92.

6. Ezuddin NS, Alawa KA, Galor A. Therapeutic strategies to treat dry eye in an aging population. Drugs Aging. 2015;32(7):505-513.

7. Tomlinson A, Khanal S. Assessment of tear film dynamics: quantification approach. Ocul Surf. 2005;3(2):81-95.

8. Hessen M, Akpek EK. Dry eye: an inflammatory ocular disease. J Ophthalmic Vis Res. 2014;9(2):240-250.

9. Downie LE, Keller PR. A pragmatic approach to the management of dry eye disease: evidence into practice. Optom Vis Sci. 2015;92(9):957-966.

10. Tiffany JM. The normal tear film. Dev Ophthalmol. 2008;41:1-20.

11. Zhou L, Beuerman RW. Tear analysis in ocular surface diseases. Prog Retin Eye Res. 2012;31(6):527-550.

12. King-Smith PE, Bailey MD, Braun RJ. Four characteristics and a model of an effective tear film lipid layer (TFLL). Ocul Surf. 2013;11(4): 236-245.

13. Bron AJ, Tiffany JM, Gouveia SM, Yokoi N, Voon LW. Functional aspects of the tear film lipid layer. Exp Eye Res. 2004;78(3):347-360.

14. Moshirfar M, Pierson K, Hanamaikai K, Santiago-Caban L, Muthappan V, Passi SF. Artificial tears potpourri: a literature review. Clin Ophthalmol. 2014;8:1419-1433.
Clinical Ophthalmology

\section{Publish your work in this journal}

Clinical Ophthalmology is an international, peer-reviewed journal covering all subspecialties within ophthalmology. Key topics include: Optometry; Visual science; Pharmacology and drug therapy in eye diseases; Basic Sciences; Primary and Secondary eye care; Patient Safety and Quality of Care Improvements. This journal is indexed on

\section{Dovepress}

PubMed Central and CAS, and is the official journal of The Society of Clinical Ophthalmology (SCO). The manuscript management system is completely online and includes a very quick and fair peer-review system, which is all easy to use. Visit http://www.dovepress.com/ testimonials.php to read real quotes from published authors. 